\title{
Abemaciclib Mesylate
}

National Cancer Institute

\section{Source}

National Cancer Institute. Abemaciclib Mesylate. NCI Thesaurus. Code C162507.

The mesylate salt of abemaciclib, an orally available cyclin-dependent kinase (CDK) inhibitor that targets the cyclin D1-CDK4 and cyclin D3-CDK6 cell cycle pathway, with potential antineoplastic activity. Abemaciclib specifically inhibits CDK4 and 6, thereby inhibiting retinoblastoma (Rb) protein phosphorylation in early G1. Inhibition of Rb phosphorylation prevents CDK-mediated G1-S phase transition, thereby arresting the cell cycle in the G1 phase, suppressing DNA synthesis and inhibiting cancer cell growth. Overexpression of the serine/threonine kinases CDK4/6, as seen in certain types of cancer, causes cell cycle deregulation. 